Dundee Dental

Education Centre

A $\mathfrak{3} 3$ million state-of-the-art dental facility has recently been opened in Dundee.

The Dundee Dental Education Centre provides a flagship facility and a focus for education and training for dentistry in the East of Scotland. The Centre has been created within the Frankland Building on the University of Dundee campus, which is now joined by a physical link to the neighbouring Dundee Dental Hospital and School.

The facility will provide postgraduate dental education and clinical skills training for all dental professionals and will also support preregistration training for dental nurses as well as undergraduate education.

The most striking feature in the new centre

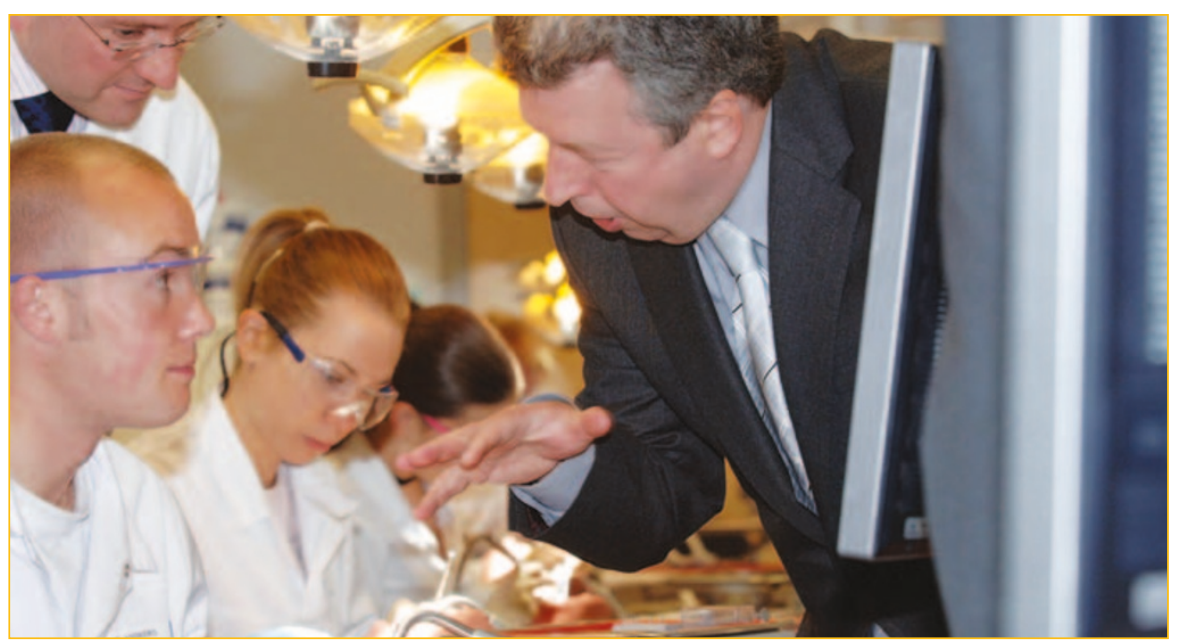

\section{Yo ho ho and a bottle of mouthwash}

Staff at the Dartford Road Dental Centre in West Dartford took part in an annual 'Dress Up Day' on behalf of the dental charity Dentaid recently, to raise funds for much needed dental and oral health care in the developing world. The Dental Centre was decorated with a 'Pirates of the Caribbean' theme. The idea came from dental nurse Beverley Bushell, who coordinated the make up and costumes, while centre manager Kim Bennett explained the purpose of the day to all the worried looking clients and children. is a 'phantom heads' clinical skills area for both postgraduate and undergraduate training. This unit comprises 38 fixed student simulators with phantom heads and fully integrated audiovisual facilities, allowing advanced hands-on training.

Mrs Ann Markham OBE, Chair of NHS Education for Scotland said, 'Dentists and dental care professionals will benefit from training which is based on the quality evidence-based guidelines which are being produced by the Dental Clinical Effectiveness Group based at the centre. Tutors there will use these guidelines, along with sound educational principles, to deliver training to an expanding client base.'

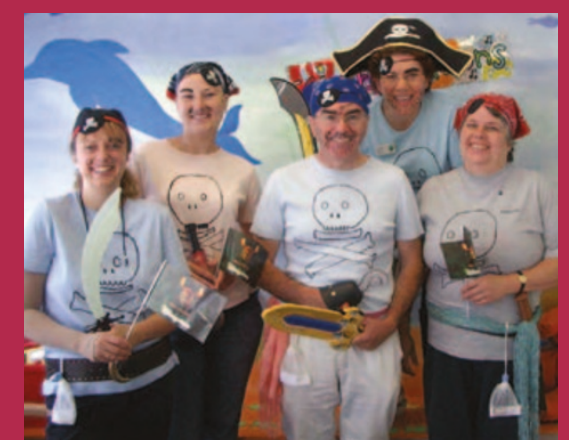

From left to right: Jolly Roger, Long John Silver, Bluebeard, Hook and Smee.

\section{Beautiful molecules}

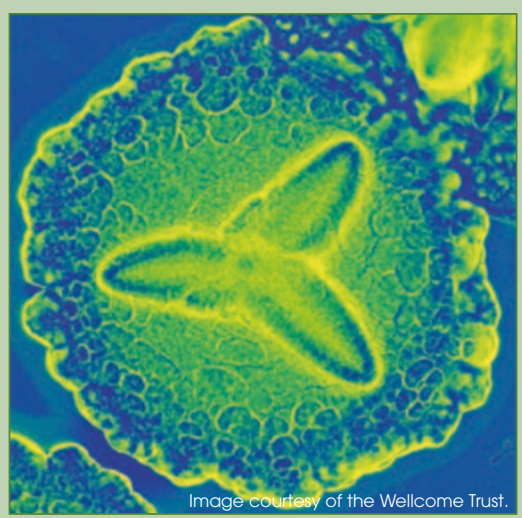

An image of an oral bacterium was amongst a number of stunning images honoured at the Wellcome Trust Biomedical Image Awards 2006. The image, actinobacillus actinomycetemcomitans, was captured by Derren Ready from the Eastman Dental Institute, London.

Other winning images included the blind spot of an eye, the stinging hairs on a nettle leaf and cancerous cells.

The winning images were selected from those submitted to the Medical Photographic Library over the past 12 months. This collection of over 160,000 images covers modern medicine and biomedical science, the history of medicine and the history of human culture from the earliest periods of civilisation to the present day, reflecting the diversity of the interests of Sir Henry Wellcome.

Other images recognised in the awards ceremony included human colon cancer cells, taken by Dave McCarthy and Annie Cavanagh from the University of London School of Pharmacy and squamous cell carcinoma, taken by Anne Weston from Cancer Research UK. The latter shows a cell from the second most common form of skin cancer, frozen and split open to reveal its nucleus, an aggressive, beastlike cell.

The award winning images have been on display at the Wellcome Library, 210 Euston Road, London, since 13 July, along with audio clips of the scientists behind the images discussing their work. Entrance to the exhibition is free. They can also be viewed online at www. wellcome.ac.uk/bia2006. 